BMJ Open Diabetes Research \& Care

\title{
Association of TCF7L2 variation with single islet autoantibody expression in children with type 1 diabetes
}

\author{
Maria J Redondo, ${ }^{1}$ Jesse Muniz, ${ }^{2}$ Luisa M Rodriguez, ${ }^{1}$ Dinakar lyer, ${ }^{3}$ \\ Fariba Vaziri-Sani, ${ }^{4}$ Morey W Haymond, ${ }^{5}$ Christiane S Hampe, ${ }^{6}$ Michael L Metzker, ${ }^{2}$ \\ Struan F A Grant, ${ }^{7}$ Ashok Balasubramanyam ${ }^{3}$
}

To cite: Redondo MJ, Muniz J, Rodriguez LM, et al. Association of TCF7L2 variation with single islet autoantibody expression in children with type 1 diabetes. BMJ Open Diabetes Research and Care 2014;2: e000008. doi:10.1136/ bmjdrc-2013-000008

Received 29 November 2013 Revised 16 January 2014 Accepted 13 February 2014

\section{CrossMark}

For numbered affiliations see end of article.

Correspondence to Dr Maria J Redondo; redondo@bcm.edu

\section{ABSTRACT}

Background: The transcription factor 7-like 2 (TCF7L2) gene has the strongest genetic association with type 2 diabetes. TCF7L2 also associates with latent autoimmune diabetes in adults, which often presents with a single islet autoantibody, but not with classical type 1 diabetes.

Methods: We aimed to test if TCF7L2 is associated with single islet autoantibody expression in pediatric type 1 diabetes. We studied 71 prospectively recruited children who had newly diagnosed type 1 diabetes and evidence of islet autoimmunity, that is, expressed $\geq 1$ islet autoantibody to insulin, glutamic acid decarboxylase 65 , islet cell autoantigen 512 , or zinc transporter 8. TCF7L2 rs7903146 alleles were identified. Data at diagnosis were cross-sectionally analyzed.

Results: We found that $21.1 \%$ of the children with autoimmune type 1 diabetes expressed a single islet autoantibody. The distribution of TCF7L2 rs7903146 genotypes in children with a single autoantibody $(\mathrm{n}=15)$ was $40 \%$ CC, $26.7 \%$ CT and $33.3 \%$ TT, compared with children with $\geq 2$ islet autoantibodies (50\% CC, $42.9 \%$ CT and $7.1 \%$ TT, $\mathrm{p}=0.024)$. Furthermore, compared with children with $\geq 2$ autoantibodies, single-autoantibody children had characteristics reflecting milder autoimmune destruction of $\beta$-cells. Restricting to lean children (body mass index<85th centile; $n=36$ ), $45.5 \%$ of those expressing a single autoantibody were rs7903146 TT homozygotes, compared with $0 \%$ of those with $\geq 2$ autoantibodies $(p<0.0001)$.

Conclusion: These results suggest that, in children with only mild islet autoimmunity, mechanisms associated with TCF7L2 genetic variation contribute to diabetogenesis, and this contribution is larger in the absence of obesity.

\section{INTRODUCTION}

Genetic variation within the transcription factor 7-like 2 (TCF7L2) gene is the most strongly associated locus with type 2 diabetes. $^{1}{ }^{2}$ The single nucleotide polymorphism (SNP) rs7903146 is considered the best variant to test due to the consistent association of the Tallele or the TT genotype with

\section{Key messages}

- Expression of single islet autoantibody was found in about $20 \%$ of children with new onset type 1 diabetes and was associated with clinical characteristics of milder autoimmune destruction of beta-cells, compared to those of children expressing $\geq 2$ autoantibodies.

- In children with type 1 diabetes and only mild islet autoimmunity, as reflected by expression of a single islet autoantibody, the TCF7L2 genetic variant that is associated with type 2 diabetes and LADA was more common than in children with type 1 diabetes and two or more positive islet autoantibodies.

- The association between the TCF7L2 genetic variant and single-autoantibody pediatric type 1 diabetes was stronger in the absence of obesity.

type 2 diabetes across multiple ethnicities; ${ }^{3}$ indeed, it is widely considered to be the causative event at this locus. ${ }^{1}$ The frequency of this variant is also increased among patients presenting with 'latent autoimmune diabetes in adults' (LADA), ${ }^{4-7}$ a form of slowly progressive autoimmune diabetes with partially preserved $\beta$-cell function and presentation in adulthood with a prolonged period of insulin independence, ${ }^{589}$ but not in patients with classical type 1 diabetes. ${ }^{10} 11$

TCF7L2 encodes a transcription factor that plays a key role in the Wnt signaling pathway. ${ }^{12}$ TCF7L2 regulates transcription of the proglucagon gene, which encodes glucagon in the pancreas, and glucagon-like peptide 1 (GLP-1) in the intestine and the brain. ${ }^{13}$ Carriers of the T allele of the rs7903146 SNP have an abnormal incretin response causing $\beta$-cell dysfunction. ${ }^{10}{ }^{14}$ In the liver, TCF7L2 risk alleles are associated with increased glucose release during fasting. ${ }^{14} 15$ The clinical interest in understanding the role of this gene is heightened because incretin dysfunction and dysregulated hepatic glucose production are amenable to treatment by available 
pharmacological agents, such as incretin enhancers and metformin, respectively.

Positivity for any of the islet autoantibodies is the most widely used parameter to define autoimmune type 1 diabetes. ${ }^{16}$ The number of circulating islet autoantibodies is a strong determinant of subsequent progression to type 1 diabetes in individuals without diabetes at risk and the general population, while expression of a single autoantibody confers only low risk of developing type 1 diabetes. ${ }^{17} 18$ Although studies on $\beta$-cell function after type 1 diabetes onset are lacking in children, particularly below age $7,{ }^{19}$ adults with LADA, who have slowly progressive loss of $\beta$-cell function after the onset of diabetes, often express a single autoantibody, ${ }^{9}{ }^{20}$ which further supports the notion that a single autoantibody expression is a marker of milder $\beta$-cell autoimmunity.

We hypothesized that patients who develop autoimmune type 1 diabetes during childhood despite only mild islet autoimmunity, as reflected by possessing a single positive islet autoantibody, are more likely to harbor the diabetogenic TCF7L2 rs7903146 TT genotype. Hence, the goal of this study was to compare, in a cohort of children with newly diagnosed autoimmune type 1 diabetes, the frequency of the rs7903146 TT genotype and clinical features among those with a single islet autoantibody and those with $\geq 2$ positive islet autoantibodies.

\section{METHODS}

Subjects

We prospectively recruited 119 children who presented with newly diagnosed diabetes at Texas Children's Hospital between October 2010 and October 2011. The study was approved by the Institutional Review Board of Baylor College of Medicine. Fasting C peptide concentrations were measured $\geq 3$ weeks after diagnosis (median $=7$ weeks, $\mathrm{SD}=3$ ) to avoid the potential effect on $\beta$-cell function of metabolic instability and diabetic ketoacidosis (DKA) that may accompany onset of disease. Insulin autoantibodies were measured within 10 days of diagnosis (prior to development of antibodies to exogenous insulin). Other islet autoantibodies-to glutamic acid decarboxylase 65 (GAD65), islet cell autoantigen 512 (ICA512/IA2) and zinc transporter 8 (ZnT8)-were measured at the 7-week (median, $\mathrm{SD}=3$ ) visit. For this cross-sectional analysis, we included only children with evidence of autoimmune diabetes to prevent including cases of type 2 diabetes, which is known to associate with the genetic variant under study. We excluded those who did not have all four islet autoantibodies measured $(n=27)$ and those who were negative for all four islet autoantibodies $(n=21)$. The final sample comprised 71 patients.

\section{Data collection}

Demographic information included date of birth, date of diagnosis, sex, and race/ethnicity. Anthropometric information included weight and height measured at the first clinical visit (mean $\pm \mathrm{SD}=10.3$ weeks \pm 5.4 ) to avoid the effect of dehydration on weight at the time of diagnosis. Tanner pubertal staging was performed by a pediatric endocrinologist. Biochemical data included glucose, glycated hemoglobin (HbAlc), $\mathrm{pH}$, bicarbonate, and $\beta$-hydroxybutyrate measured at diagnosis. Body mass index (BMI) was calculated in children older than 2 years based on height and weight and was categorized using sex-specific and age-specific percentiles by Centers for Disease Control and Prevention criteria. ${ }^{21}$ Obesity was defined as BMI at or above the 95th sex-specific and age-specific BMI centile, and overweight as BMI centile $\geq 85$ th to $<95$ th. In children below age 2 years $(n=11)$, BMI was considered a missing value because of the lack of standardized age-adjusted and gender-adjusted BMI data for ages $0-2$ years. DKA was defined by venous blood $\mathrm{pH}<7.3$ and bicarbonate $<15 \mathrm{mEq} / \mathrm{L}$.

\section{Laboratory methods}

GAD65, ICA512/IA2, and ZnT8 autoantibodies were measured by the radioligand binding assay as previously described. $^{22} 23$ Samples were considered ICA512/IA2 autoantibody positive if binding exceeded that of the 98th centile for healthy controls $(30 \mathrm{RU} / \mathrm{mL})$. For ZnT8 autoantibodies, a cutoff was set at $15 \mathrm{RU} / \mathrm{mL}$ for autoantibodies for ZnT8Arg and $26 \mathrm{RU} / \mathrm{mL}$ for ZnT8Trp based on the 98th centile observed in 50 healthy human control sera. Samples were considered ZnT8 autoantibody positive if binding to either ZnT8Arg or ZnT8Trp was detected. Our laboratory participated in the Diabetes Autoantibody Standardization Program (DASP) ${ }^{24}$ workshop and the GAD65 autoantibody assay showed $86 \%$ sensitivity and $93 \%$ specificity, and the ICA512/IA2 autoantibody assay showed $66 \%$ sensitivity and 98\% specificity. ZnT8 autoantibodies were not included in the workshop. Insulin autoantibodies were measured by the Quest Diagnostics Nichols Institute (San Juan Capistrano, California, USA) by radioimmunoassay (RIA) with clinical sensitivity and specificity of $50 \%$ and $99 \%$, respectively (positivity $>0.4 \mathrm{U} /$ $\mathrm{mL}$ ). Serum $\mathrm{C}$ peptide was measured by highly specific RIA (Human C Peptide RIA kit, Millipore Research Inc, St Louis, Missouri, USA).

\section{Genetic methods}

Genomic DNA was extracted from peripheral blood leukocytes by standard methods as previously described. ${ }^{25}$ Multiple displacement amplification (MDA) of genomic DNA, PCR, and direct DNA sequencing were performed as previously described. ${ }^{26}$ Heterozygous bases were called if the minor peak was $\geq 25 \%$ the height of the major peak. TaqMan assays: Approximately 10-100 ng MDA DNA was used as the template according to the manufacturer's protocol. For the TCF7L2 rs7903146 allele, we designed primers and probe using the Life Technologies custom TaqMan assay website (5'-CCTCA AACCTAGCACAGCTGTTAT [forward primer], 5'-TGAA 
AACTAAGGGTGCCTCATACG [reverse primer], and $5^{\prime}$-CTTTTTAGATA[C/T]TATATAATTTAA [probe]) and confirmed those results by direct DNA sequencing. Because the rs7903146 allele can be flanked by an 8-basepair insertion/deletion (indel) polymorphisms on its $5^{\prime}$-end and a 1-base-pair indel on its 3 '-end, heterozygous base calls were confirmed by $\geq 3$ single direction reads in the contig. For $D Q B 1^{*} 0302$, the rs 7454108 allele was analyzed using primers and probe described by Barker et $a .^{27}$ Allele frequencies were calculated from multiple sequence alignments.

\section{Statistical analysis}

Statistical analyses were conducted with STATA V.10 (StataCorp 2007, Stata Statistical Software: Release 10. College Station, Texas: StataCorp LP). Genotype distribution in the study sample was in the Hardy-Weinberg equilibrium. To compare proportions among groups, we used $\chi^{2}$ test. To compare means we used $\mathrm{t}$ tests, Mann-Whitney U tests, or one-way analysis of variance (ANOVA) where appropriate. The Kruskal-Wallis rank test was used to compare rank distributions of a continuous variable across categories of another variable when the conditions for analysis of variance were not met (eg, unequal variances). Fasting serum C peptide was $\log$ transformed due to the non-normality of its distribution; elevated C-peptide was defined as values greater than the median. Sample size estimation was not performed a priori for this pilot study, but rather all available cases were used. Throughout the manuscript, data are expressed as mean $\pm \mathrm{SD}$ unless otherwise stated. $\mathrm{p}$ Values $\leq 0.05$ were considered statistically significant.

\section{RESULTS}

Mean age of the 71 children at diagnosis of autoimmune type 1 diabetes was $10.7 \pm 3.8$ years. A single autoantibody was expressed by $15(21.1 \%)$ of the children. Other demographic and clinical characteristics are depicted in table 1 .

The distribution of TCF7L2 rs7903146 genotypes in children with a single islet autoantibody $(\mathrm{n}=15)$ was significantly different from that of children with $\geq 2$ islet autoantibodies, as illustrated in table 2 . The minor allele frequencies were $46.7 \%$ and $28.6 \%$, respectively.

Furthermore, as illustrated in table 2, children with a single positive autoantibody were less likely than children with $\geq 2$ islet autoantibodies to express autoantibodies to ICA512/IA2 or ZnT8, while there were no statistical differences for GAD65 or insulin autoantibodies. Titers of ICA512/IA2 and Znt8Arg were significantly lower among children with a single autoantibody. Children who expressed a single islet autoantibody had lower frequency of DKA, lower serum concentrations of $\beta$-hydroxybutyrate at diagnosis, and were more likely to have elevated fasting $\mathrm{C}$ peptide levels (defined as values above the median of the logarithmic distribution of $\mathrm{C}$ peptide; figure 1 ).
Table 1 Characteristics of children at diagnosis of autoimmune type 1 diabetes $(\mathrm{N}=71)$

\begin{tabular}{ll}
\hline Age (years): mean (SD) & $10.7 \pm 3.8$ \\
Male gender: $n$ (\%) & $43(60.6)$ \\
Race/ethnicity: $n$ (\%) & $39(54.9)$ \\
$\quad$ Non-Hispanic white & $23(32.4)$ \\
Hispanic & $6(8.5)$ \\
African-American & $3(4.2)$ \\
Other & $76.2 \pm 22.3$ \\
BMI percentile: mean (SD) & $34(48.6)$ \\
Obesity or overweight: $n$ (\%) & $21.8 \pm 10.2$ \\
Glucose (mmol/L): mean (SD) & $12.1 \pm 2.2(109 \pm 24)$ \\
HbA1c (\%) (mmol/mol): mean (SD) & $26(38.8)$ \\
DKA: $n$ (\%) & $5.3 \pm 4.3$ \\
Beta-hydroxybutyrate (mmol/L): & \\
mean (SD) & $0.143(0.083-0.243)$ \\
Fasting C peptide (nmol/L): & \\
median (25th-75th centiles) & \\
Expression of islet & \\
autoantibodies: $n$ (\%) & $27(38)$ \\
Insulin & $41(57.8)$ \\
GAD65 & $52(73.2)$ \\
ICA512/IA2 & $44(62)$ \\
ZnT8 & \\
Number of islet autoantibodies & \\
expressed: $n$ (\%) & \\
1 & $15(21.1)$ \\
2 & $25(35.2)$ \\
3 & $25(35.2)$ \\
4 & $6(8.5)$ \\
HLA DR3-DQ2/DR4-DQ8: $n$ (\%) & $16(22.5)$ \\
\hline BMl, body mass index; DKA, diabetic ketoacidosis; GAD65, \\
glutamic acid decarboxylase 65; HbA1c, glycated hemoglobin; \\
HLA, human leukocyte antigen; ICA512//A2, islet cell autoantigen \\
$512 ;$ ZnT8, zinc transporter 8. &
\end{tabular}

Children who were lean $(\mathrm{BMI}<85$ th centile $)$ and expressed a single islet autoantibody $(\mathrm{n}=11)$ had a significantly higher frequency of the TCF7L2 TT genotype (45.5\%) compared with lean children with $\geq 2$ positive autoantibodies; in the latter group, this genotype was completely absent $(\mathrm{n}=25,0 \%, \mathrm{p}<0.0001)$. Lean children with a single autoantibody were also more likely to have an elevated $\mathrm{C}$ peptide ( $72.7 \%$ vs $32.0 \%, \mathrm{p}=0.023)$ and less likely to express ICA512/IA2 ( $\mathrm{p}<0.0001)$, ZnT8 ( $\mathrm{p}=0.01)$, and GAD65 autoantibodies $(\mathrm{p}=0.04)$ compared with lean children who expressed $\geq 2$ autoantibodies. They tended to present less often with DKA ( $10 \%$ vs $39.1 \%, \mathrm{p}=0.094$ ) and had lower levels of $\beta$-hydroxybutyrate $(\mathrm{p}<0.03)$.

Among obese or overweight children $(n=34)$, those with a single and $\geq 2$ islet autoantibodies were not statistically different for TT genotype frequency $(0 \%$ and $13.3 \%$, respectively, $\mathrm{p}=0.44), \mathrm{C}$ peptide, DKA at onset, $\beta$-hydroxybutyrate, or expression of GAD65 or insulin autoantibodies. Expression of ICA512/IA2 autoantibodies was lower $(\mathrm{p}<0.001)$, and that of ZnT8 autoantibodies tended to be lower $(\mathrm{p}=0.07)$ in single compared with $\geq 2$ autoantibody-positive obese type 1 diabetes children. 
Table 2 Comparison of children with a single and $\geq 2$ positive islet autoantibodies at diagnosis of type 1 diabetes

\begin{tabular}{|c|c|c|c|}
\hline & $\begin{array}{l}\text { Single positive } \\
\text { autoantibody } \\
\mathrm{N}=15\end{array}$ & $\begin{array}{l}\geq 2 \text { Positive } \\
\text { autoantibodies } \\
\mathrm{N}=56\end{array}$ & p Value \\
\hline Age (years): mean (SD) & $11.7 \pm 4.6$ & $10.4 \pm 3.6$ & 0.25 \\
\hline Male gender: $n(\%)$ & $9(60)$ & $34(60.7)$ & 0.96 \\
\hline Race/ethnicity: n (\%) & & & 0.89 \\
\hline Non-Hispanic white & $9(60)$ & $30(53.6)$ & \\
\hline Hispanic & $4(26.7)$ & 19 (33.9) & \\
\hline African-American & $1(6.7)$ & $5(8.9)$ & \\
\hline Other & $1(6.7)$ & $2(3.6)$ & \\
\hline BMI centile: mean (SD) & $64.1 \pm 25.3$ & $79.7 \pm 20.41$ & 0.01 \\
\hline Obesity or overweight: $n(\%)$ & $4(26.7)$ & $30(54.6)$ & 0.06 \\
\hline Glucose (mmol/L): mean (SD) & $19.2 \pm 5.5$ & $22.5 \pm 11.1$ & 0.26 \\
\hline $\mathrm{HbA1c}(\%)$ (mmol/mol): mean (SD) & $12.6 \pm 2(114 \pm 21.9)$ & $11.9 \pm 2.2(107 \pm 24)$ & 0.29 \\
\hline DKA: n (\%) & $2(14.3)$ & $24(45.3)$ & 0.034 \\
\hline$\beta$-hydroxybutyrate (mmol/L): mean (SD) & $2.6 \pm 2.3$ & $6 \pm 4.4$ & 0.006 \\
\hline Elevated $^{*}$ fasting $\mathrm{C}$ peptide: $\mathrm{n}(\%)$ & $11(73.3)$ & $24(42.9)$ & 0.036 \\
\hline Fasting C peptide (ng/mL): median (IQR) & $1.19(0.76)$ & $0.67(1.5)$ & 0.96 \\
\hline \multicolumn{4}{|l|}{ Positive islet autoantibodies: $\mathrm{n}(\%)$} \\
\hline Insulin & $4(26.7)$ & $23(41.1)$ & 0.31 \\
\hline GAD65 & $6(40)$ & $35(62.5)$ & 0.12 \\
\hline ICA512/IA2 & $2(13.3)$ & $50(89.3)$ & 0.0001 \\
\hline ZnT8 & $3(20)$ & 41 (73.2) & 0.0001 \\
\hline \multicolumn{4}{|l|}{ Titers of islet autoantibodies: mean (SD) } \\
\hline Insulin & $0.46(0.487)$ & $1.617(5.295)$ & 0.4 \\
\hline GAD65 & $0.152(0.256)$ & $0.37(0.603)$ & 0.18 \\
\hline ICA512/IA2 & $0.115(0.298)$ & $0.617(0.421)$ & 0.0001 \\
\hline ZnT8Arg & $19.6(51.4)$ & $161(239.1)$ & 0.027 \\
\hline ZnT8Trp & $10.8(24.6)$ & $139.6(263.6)$ & 0.064 \\
\hline HLA DR3-DQ2/DR4-DQ8: n (\%) & $3(20)$ & $13(23.2)$ & 0.79 \\
\hline TCF7L2 SNP rs7903146 genotype: n (\%) & & & 0.024 \\
\hline $\mathrm{CC}$ & $6(40)$ & $28(50)$ & \\
\hline CT & $4(26.7)$ & $24(42.9)$ & \\
\hline $\mathrm{TT}$ & $5(33.3)$ & $4(7.1)$ & \\
\hline
\end{tabular}

*Above the median of the logarithmic distribution.

BMI, body mass index; DKA, diabetic ketoacidosis; GAD65, glutamic acid decarboxylase 65; HbA1c, hemoglobin A1c; HLA, human leukocyte antigen; ICA512/IA2, islet cell autoantigen 512; IQR, interquartile range; SNP, single nucleotide polymorphism; TCF7L2, transcription factor 7-like 2; ZnT8, zinc transporter 8.

\section{DISCUSSION}

In the present study, we observed that the TCF7L2 locus is associated with single islet autoantibody expression in children diagnosed with autoimmune type 1 diabetes. Children with single autoantibody positivity were less likely to express ICA512/IA2 and ZnT8 autoantibodies, and had lower incidence of DKA and higher fasting $\mathrm{C}$ peptide levels than children with $\geq 2$ positive autoantibodies. When the cohort was divided into lean and obese/overweight subgroups, most of these differences were present in the lean children but not among overweight or obese children. Almost half of the lean children with a single islet autoantibody were rs7903146 TT homozygotes, compared to none of the lean children with $\geq 2$ positive autoantibodies.

Children with single islet autoantibody positivity had additional evidence of milder islet autoimmunity. They were less likely to present with DKA and had lower plasma concentration of $\beta$-hydroxybutyrate at diagnosis than children with $\geq 2$ islet autoantibodies. Since DKA is a marker of nearcomplete insulin deficiency, these findings are consistent with less aggressive $\beta$-cell destruction. Indeed, compared with children with $\geq 2$ positive autoantibodies, those with a single autoantibody had greater $\beta$-cell function, as measured by fasting serum $\mathrm{C}$ peptide. Single-autoantibody children were also less likely to express ICA512/IA2 and ZnT8 autoantibodies. Seroconversion of ICA512/IA2 and ZnT8 autoantibodies has been found to immediately precede progression to type 1 diabetes in subjects at risk, ${ }^{28} 29$ reflecting their association with aggressiveness of the autoimmune attack on $\beta$-cells.

Our finding that children with type 1 diabetes and mild islet autoimmunity were more likely to be carriers of the TCF7L2 rs7903146 TT genotype suggests that a TCF7L2-dependent mechanism may be contributing to their diabetes. The TCF7L2 protein is expressed in the $\beta$-cell and is involved in proliferation and apoptosis, insulin processing, and regulated expression of receptors 


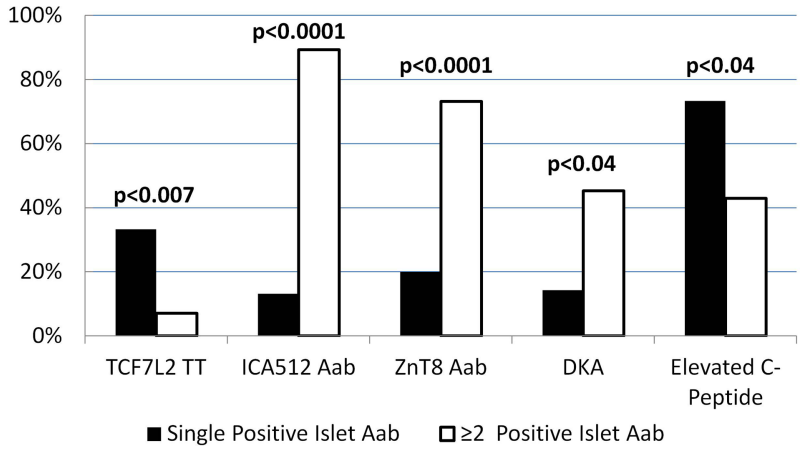

Figure 1 Percentages of children with autoimmune type 1 diabetes who carried the TCF7L2 rs7903146 TT genotype, expressed ICA512/IA2 or ZnT8 autoantibodies, presented with DKA and had elevated fasting $C$ peptide levels 7 weeks after onset: comparison with children with a single versus $\geq 2$ positive islet autoantibodies. Black columns=children expressing a single islet autoantibody. White columns= children expressing $\geq 2$ positive islet autoantibodies. Aab, autoantibody; DKA, diabetic ketoacidosis; ICA512, islet cell autoantigen 512; TCF7L2, transcription factor 7-like 2; ZnT8, zinc transporter 8.

to glucose-dependent insulinotropic polypeptide and GLP-1 on the $\beta$-cell. ${ }^{10}{ }^{14}$ In healthy subjects and patients with type 2 diabetes, TCF7L2 gene variants are associated with decreased insulin secretion, likely through an impaired incretin effect. ${ }^{30}$ TCF7L2-regulated incretins may also have effects on the brain and thus potentially on appetite regulation. ${ }^{13}$ The TCF7L2 gene also regulates hepatic glucose release during fasting. ${ }^{14} 15$

We speculate that several diabetogenic mechanisms, for example, autoimmune loss of $\beta$-cells, nonautoimmune $\beta$-cell dysfunction (eg, influenced by TCF7L2 genetic status), and insulin resistance may simultaneously contribute to development of diabetes in the same individual. LADA and now pediatric diabetes with a single autoantibody and the TCF7L2 rs7903146 TT genotype are examples of variable contributions of multiple pathogenic pathways leading to clinically distinct forms of diabetes. Supporting this hypothesis, the association of the diabetogenic TCF7L2 variant with single islet autoantibody expression was observed only in lean children, while it was not present in overweight or obese children. A plausible explanation for this observation is that, in lean children with mild autoimmunity, an additional factor, such as determined by TCF7L2 status, was necessary for development of diabetes. On the other hand, only mild autoimmune $\beta$-cell loss was sufficient to cause diabetes in obese children, possibly because of the elevated demand for insulin imposed by obesity-induced insulin resistance. These hypotheses are also consistent with a previous report of lower BMI in adults with slowly progressive forms of autoimmune diabetes or type 2 diabetes who carried the diabetogenic TCF7L2 variant, compared with non-carriers. ${ }^{4}$

To our knowledge, this is the first study on the prevalence of the TCF7L2 rs7903146 $\mathrm{T}$ allele in subsets of children with autoimmune type 1 diabetes and, in particular, in relation to the severity of islet autoimmunity. Previous pediatric studies have reported that the type 2 diabetes-associated TCF7L2 gene polymorphism is more frequent in diabetes without evidence of islet autoimmunity, for example, in autoantibody-negative than autoantibody-positive type 1 diabetes children ${ }^{30}$ and in GAD65 autoantibody-negative than GAD65 autoantibody-positive patients aged $15-34 .^{31}$ In contrast, we studied the contribution of TCF7L2 in well-defined subsets of children with autoimmune diabetes. One limitation of this study is the relatively small sample size, which may affect the generalizability of the study. Confirmation of our findings in an independent study sample is warranted. Another limitation is the use of expression of a single islet autoantibody to define mild islet autoimmunity. Although in our study the expression of a single islet autoantibody correlated with clinical and laboratory indicators of mild $\beta$-cell autoimmune destruction, the identification of patients with mild islet autoimmunity may improve as the sensitivity and specificity of current autoantibody assays increase, and as better markers of the aggressiveness of the immune attack on $\beta$-cells are validated.

In summary, our findings that the type 2 diabetesassociated TCF7L2 variant is carried in the homozygote form by a third of children with type 1 diabetes and single islet autoantibody positivity (and almost half of the lean children with type 1 diabetes and a single islet autoantibody) support the hypothesis that nonautoimmune pathways may significantly contribute to diabetogenesis in well-defined subsets of pediatric autoimmune type 1 diabetes.

\section{Author affiliations}

${ }^{1}$ Department of Pediatrics, Section of Diabetes and Endocrinology, Texas Children's Hospital, Baylor College of Medicine, Houston, Texas, USA

${ }^{2}$ Department of Genetics, Human Genome Center, Baylor College of Medicine, Houston, Texas, USA

${ }^{3}$ Division of Diabetes, Translational Metabolism Unit, Diabetes Research Center, Endocrinology and Metabolism, Baylor College of Medicine, Houston, Texas, USA

${ }^{4}$ Department of Clinical Sciences, Diabetes and Celiac Disease, Lund University/CRC, Malmö, Sweden

${ }^{5}$ Childrens's Nutrition Research Center, Baylor College of Medicine, Houston, Texas, USA

${ }^{6}$ Department of Medicine, University of Washington, Seattle, Washington, USA ${ }^{7}$ Division of Human Genetics and Center for Applied Genomics, The Children's Hospital of Philadelphia, Philadelphia, Pennsylvania, USA

Correction notice The Funding statement in this article has been corrected since published.

Acknowledgements The authors would like to acknowledge the statistical advice provided by Dr O'Brian Smith, and the assistance of Ms Andrene McDonald (Research Coordinator), Baylor College of Medicine, Section of Pediatric Diabetes and Endocrinology, Houston, Texas.

Contributors MJR designed and conducted the study, analyzed and interpreted the data, and wrote the first draft of the manuscript. JM conducted genetic testing and reviewed the manuscript. LMR, MWH, and AB contributed to study design, data interpretation, and review of the manuscript. DI processed the serum and DNA samples, conducted $C$ peptide testing, and reviewed the manuscript. FVS provided the ZnT8 construct and reviewed the 
manuscript. CSH oversaw autoantibody testing and contributed to data interpretation and review of the manuscript. MLM oversaw genetic testing and contributed to data interpretation and review of the manuscript.

SFAG contributed to data interpretation and review of the manuscript. MJR is the guarantor.

Funding This study was made possible by a Pediatric Pilot Award grant from Texas Children' Hospital (MJR), and R01 DK085212 (SFAG).

Competing interests None.

Patient consent Obtained.

Ethics approval Baylor College of Medicine IRB.

Provenance and peer review Not commissioned; externally peer reviewed.

Data sharing statement No additional data are available.

Open Access This is an Open Access article distributed in accordance with the Creative Commons Attribution Non Commercial (CC BY-NC 3.0) license, which permits others to distribute, remix, adapt, build upon this work noncommercially, and license their derivative works on different terms, provided the original work is properly cited and the use is non-commercial. See: http:// creativecommons.org/licenses/by-nc/3.0/

\section{REFERENCES}

1. Grant SF, Thorleifsson G, Reynisdottir I, et al. Variant of transcription factor 7-like 2 (TCF7L2) gene confers risk of type 2 diabetes. Nat Genet 2006:38:320-3.

2. Bonetti S, Trombetta M, Malerba G, et al. Variants and haplotypes of TCF7L2 are associated with $\beta$-cell function in patients with newly diagnosed type 2 diabetes: the Verona Newly Diagnosed Type 2 Diabetes Study (VNDS) 1. J Clin Endocrinol Metab 2011;96:E389-93.

3. Cauchi S, El Achhab Y, Choquet H, et al. TCF7L2 is reproducibly associated with type 2 diabetes in various ethnic groups: a global meta-analysis. J Mol Med 2007;85:777-82.

4. Lukacs K, Hosszufalusi N, Dinya $\mathrm{E}$, et al. The type 2 diabetes-associated variant in TCF7L2 is associated with latent autoimmune diabetes in adult Europeans and the gene effect is modified by obesity: a meta-analysis and an individual study. Diabetologia 2012;55:689-93.

5. Cervin C, Lyssenko V, Bakhtadze E, et al. Genetic similarities between latent autoimmune diabetes in adults, type 1 diabetes, and type 2 diabetes. Diabetes 2008;57:1433-7.

6. Groop LC, Bottazzo GF, Doniach D. Islet cell antibodies identify latent type I diabetes in patients aged 35-75 years at diagnosis. Diabetes 1986;35:237-41.

7. Zampetti S, Spoletini M, Petrone A, et al. Association of TCF7L2 gene variants with low GAD autoantibody titre in LADA subjects (NIRAD Study 5). Diabet Med 2010;27:701-4.

8. Tuomi T, Groop LC, Zimmet PZ, et al. Antibodies to glutamic acid decarboxylase reveal latent autoimmune diabetes mellitus in adults with a non-insulin-dependent onset of disease. Diabetes 1993;42:359-62.

9. Pozzilli P, Di Mario U. Autoimmune diabetes not requiring insulin at diagnosis (latent autoimmune diabetes of the adult): definition, characterization, and potential prevention. Diabetes Care 2001;24:1460-7.

10. Qu HQ, Polychronakos C. The TCF7L2 locus and type 1 diabetes. BMC Med Genet 2007;8:51.

11. Raj SM, Howson JM, Walker NM, et al. No association of multiple type 2 diabetes loci with type 1 diabetes. Diabetologia 2009;52:2109-16.
12. Prunier C, Hocevar BA, Howe PH. Wnt signaling: physiology and pathology. Growth Factors 2004;22:141-50.

13. Shao W, Wang D, Chiang YT, et al. The Wnt signaling pathway effector TCF7L2 controls gut and brain proglucagon gene expression and glucose homeostasis. Diabetes 2013;62:789-800.

14. Lyssenko V, Lupi R, Marchetti P, et al. Mechanisms by which common variants in the TCF7L2 gene increase risk of type 2 diabetes. J Clin Invest 2007;117:2155-63.

15. Boj SF, van Es JH, Huch M, et al. Diabetes risk gene and Wnt effector Tcf7/2/TCF4 controls hepatic response to perinatal and adult metabolic demand. Cell 2012;151:1595-607.

16. American Diabetes Association. Diagnosis and classification of diabetes mellitus. Diabetes Care 2013;36(Suppl 1):S67-74.

17. Bingley PJ, Christie MR, Bonifacio E, et al. Combined analysis of autoantibodies improves prediction of IDDM in islet cell antibodypositive relatives. Diabetes 1994;43:1304-10.

18. Yu L, Boulware DC, Beam CA, et al. Zinc transporter-8 autoantibodies improve prediction of type 1 diabetes in relatives positive for the standard biochemical autoantibodies. Diabetes Care 2012:35:1213-18.

19. Greenbaum CJ, Beam CA, Boulware D, et al. Fall in C-peptide during first 2 years from diagnosis: evidence of at least two distinct phases from composite Type 1 Diabetes TrialNet data. Diabetes 2012;61:2066-73.

20. Leslie RD, Kolb H, Schloot NC, et al. Diabetes classification: grey zones, sound and smoke: action LADA 1. Diabetes Metab Res Rev 2008;24:511-19.

21. Kuczmarski RJ, Ogden CL, Grummer-Strawn LM, et al. CDC growth charts: United States. Adv Data 2000;314:1-27.

22. Oak S, Gilliam LK, Landin-Olsson M, et al. The lack of anti-idiotypic antibodies, not the presence of the corresponding autoantibodies to glutamate decarboxylase, defines type 1 diabetes. Proc Natl Acad Sci USA 2008;105:5471-6.

23. Vaziri-Sani F, Oak S, Radtke J, et al. ZnT8 autoantibody titers in type 1 diabetes patients decline rapidly after clinical onset. Autoimmunity 2010;43:598-606.

24. Bingley PJ, Bonifacio E, Mueller PW. Diabetes antibody standardization program: first assay proficiency evaluation. Diabetes 2003;52:1128-36.

25. Maldonado M, Hampe CS, Gaur LK, et al. Ketosis-prone diabetes: dissection of a heterogeneous syndrome using an immunogenetic and beta-cell functional classification, prospective analysis, and clinical outcomes. J Clin Endocrinol Metab 2003;88: 5090-8.

26. Haaland WC, Scaduto DI, Maldonado MR, et al. A-beta-subtype of ketosis-prone diabetes is not predominantly a monogenic diabetic syndrome. Diabetes care 2009;32:873-7.

27. Barker JM, Triolo TM, Aly TA, et al. Two single nucleotide polymorphisms identify the highest-risk diabetes HLA genotype: potential for rapid screening. Diabetes 2008;57:3152-5.

28. Sosenko JM, Skyler JS, Palmer JP, et al. A longitudinal study of GAD65 and ICA512 autoantibodies during the progression to type 1 diabetes in Diabetes Prevention Trial-Type 1 (DPT-1) participants. Diabetes Care 2011;34:2435-7.

29. Long AE, Gillespie KM, Rokni S, et al. Rising incidence of type 1 diabetes is associated with altered immunophenotype at diagnosis. Diabetes 2012;61:683-6.

30. Yu J, Steck AK, Babu S, et al. Single nucleotide transcription factor 7-like 2 (TCF7L2) gene polymorphisms in antiislet autoantibodynegative patients at onset of diabetes. J Clin Endocrinol Metab 2009;94:504-10.

31. Bakhtadze E, Cervin C, Lindholm E, et al. Common variants in the TCF7L2 gene help to differentiate autoimmune from non-autoimmune diabetes in young (15-34 years) but not in middle-aged (40-59 years) diabetic patients. Diabetologia 2008;51:2224-32. 